\title{
Magnetic phase diagram of superantiferromagnetic $\mathrm{TbCu}_{2}$ nanoparticles
}

C. Echevarria-Bonet, J. I. Espeso, J. Rodríguez Fernández, M. de la Fuente Rodríguez, and L. Fernández Barquín Depto. CITIMAC, Universidad de Cantabria, 39005 Santander, Spain

$$
\text { D. P. Rojas }
$$

Depto. Estructuras y Física de la Edificación-ETSAM, Universidad Politécnica de Madrid, 28040 Madrid, Spain

L. Rodríguez Fernández

SERMET, Universidad de Cantabria, 39005 Santander, Spain

P. Gorria and J. A. Blanco

Depto. Física, Universidad de Oviedo, 33007 Oviedo, Spain

M. L. Fdez-Gubieda

Depto. Electricidad y Electrónica, Universidad del País Vasco (UPV/EHU), 48940 Leioa, Spain and BCMaterials, Parque Tecnológico de Zamudio, 48160 Derio, Spain

E. Bauer

Institut für Festkörperphysik, Technische Universität Wien, 1040 Wien, Austria

F. Damay

Laboratoire Léon Brillouin, CEA-SACLAY, 91191 Gif-sur-Yvette Cedex, France

(Dated: December 22, 2014)

\begin{abstract}
The structural state and static and dynamic magnetic properties of $\mathrm{TbCu}_{2}$ nanoparticles are reported. The nanoparticles were produced by mechanical milling under inert atmosphere with low milling times $\leq 15$ hours. The randomly dispersed nanoparticles as detected by TEM retain the bulk symmetry with an orthorhombic Imma lattice and $\mathrm{Tb}$ and $\mathrm{Cu}$ in $4 c$ positions. Rietveld refinements confirm that the milling produces a controlled reduction of particle sizes reaching down to $\simeq 6 \mathrm{~nm}$ and an increase of the microstrain $\simeq 0.6 \%$. The DC-susceptibility shows a reduction of the Néel transition (from $49 \mathrm{~K}$ to $43 \mathrm{~K}$ ) and a progressive increase of the spin glass peak (from 9 to $15 \mathrm{~K}$ ) in the zero field cooled magnetization with size reduction. The exchange anisotropy is very weak (bias field of $\simeq 30$ Oe) and is due to the presence of a disordered (thin) shell coupled to the antiferromagnetic core. The dynamic susceptibility evidences a critical slowing down in the spin disordered state with a tendency to increase of $z v$ and $\beta$ exponents when the size becomes smaller $(z v \simeq 6.6$ and $\beta \simeq 0.85)$. A Rietveld analysis of neutron diffraction patterns $1.8 \leq \mathrm{T} \leq 60$ $\mathrm{K}$ including the magnetic structure determination reveals that there is a reduction of the expected moment $(\simeq 80 \%)$ which must be connected to the presence of the disordered particle shell. The core magnetic structure retains the bulk antiferromagnetic arrangement. The overall interpretation is based on a superantiferromagnetic behavior which at low temperatures coexists with a canting of surface moments. We propose a novel magnetic phase diagram as a function of the particle size.
\end{abstract}

PACS numbers: 75.75-c,75.50.Lk,75.25.-j

\section{INTRODUCTION}

The research on magnetic nanoparticles (NPs) has become ubiquitous over the last years due to the rich magnetic phenomena arising from the combination of three key factors: i) magnetic single domains, ii) surface spin canting enhanced by the ultrafine size of the particles, and iii) the magnetic interactions due to the presence of other particles at distances in which dipolar and/or indirect exchange interactions may be acting. ${ }^{1,2}$ On top of this evident attraction, there is an obvious technological transfer acting as a substantial driving force, related especially to bioscience and environmental applications. ${ }^{3-7}$

The basic understanding of those i)-iii) factors is al- ready achieved. ${ }^{1,2}$ Yet, there is a great deal of attention (and a lack of quantitative evidence) on the fact that whenever the magnetic NPs increase their concentration in a given system, new global magnetic phenomena flourish. In this situation, the NPs no longer behave independently with a supeparamagnetic (SPM) relaxation. These phenomena have been successfully integrated within the term of Supermagnetism, and include three different magnetic states: a collection of frozen magnetic NPs in a superspin glass state (SSG), or the superferromagnetic (SFM) or superantiferromagnetic (SAFM) coupling between the particles. ${ }^{8} \mathrm{~A}$ vast majority of the latter field of research has been approached by analyzing the family of $3 d$-transition metal compounds 
in the form (mostly) of Fe-oxides and Fe NPs forming different nanostructures. ${ }^{9-11}$ Their production routes are various, and the basic characterization is relatively easy to achieve. From a different perspective, most of the work has been concentrated on ferromagnetic (FM), or ferrimagnetic (FiM) NPs, whereas the work with antiferromagnetic (AFM) NPs provides a valuable information of the particle shell and surface interface. ${ }^{12-15}$

In such context it is surprising that the other family of magnetic compounds based of $4 f$-elements (Rare Earth $=R$ ) has been barely analyzed at the nanoscale. It is true that efforts to improve and tailor the properties of permanent magnets have been pursued seizing the control of optimized nanometric structures. ${ }^{16}$ In metallic state, bulk magnetic $4 f$-alloys have constituted a profitable playground to study the magnetic interactions mediated by the indirect (RKKY) interaction and the effects of the crystalline electric field. ${ }^{17}$ Attention to the size effects is possible in $R X_{2}(X=$ metal) alloys and there is a dire need to deepen in such direction. ${ }^{12,18-22}$ The main advantages of studying these alloys are the strong variation of the magnetic coupling with the change of both the $R$ and the $X$ atoms, for which there is an established background in bulk alloys. ${ }^{17}$

To this end, it is recommendable to select simple $4 f$ alloys of which the bulk magnetic properties are known in detail. Equally, simple crystallographic structures are preferred (as in $R X_{2}$ ), displaying high magnetic moments easy to detect (Tb-based) and the non-trivial fact of displaying ordering temperatures below RT. The latter is not a nimble issue as it entails the study of finite size influence in the transitions, precisely related to Supermagnetism. Hence, the most outstanding works on magnetic $R X_{2}$ have been primarily focused on the characterization of, amongst others, $\mathrm{GdAl}_{2}$ and $\mathrm{TbAl}_{2}$ ferromagnets. In this sense, several reports have revealed the size-effects on the magnetic properties of $\mathrm{GdAl}_{2}{ }^{18,19}$, showing that the ferromagnetic transition $\left(T_{C} \approx 170 \mathrm{~K}\right)$ is weakened, leading to the appearance of a transition to a spin glass $(\mathrm{SG})$ at a lower, so-called, freezing temperature $\left(T_{f} \approx 65\right.$ $\mathrm{K})$. The same consequence due to the mechanical milling was found for $\mathrm{TbAl}_{2}$; the ferromagnetic transition at $T_{C}$ $=105 \mathrm{~K}$ tends to be wiped out with milling time, favoring a SG behavior, with $T_{f} \approx 45 \mathrm{~K} .{ }^{20}$

The $R$-nanomaterials mentioned above present a core with FM ordering, but size effects have also been explored in $R$-nanometric alloys with AFM interactions, such as $\mathrm{TbCu}_{2}$. A previous work accounted for the change from an AFM transition at $\mathrm{T}_{N}=48 \mathrm{~K}$ to a $\mathrm{SG}$ behavior $T_{f} \approx 15 \mathrm{~K}$ when bulk $\mathrm{TbCu}_{2}$ was mechanically milled up to $20 \mathrm{~h} .^{21}$ In bulk state, $\mathrm{TbCu}_{2}$ crystallizes in the Imma symmetry (orthorhombic, $\mathrm{CeCu}_{2}$-type crystal structure) and orders at $T_{N}=48 \mathrm{~K}$ with a collinear AFM structure. ${ }^{23}$ Seizing the particle size $(D)$ as the driving variable to induce magnetic moment rearrangements, the existence of a macroscopic AFM with a reentrant SG state in nanosized $\mathrm{TbCu}_{2}$ (for milling times, $t$, below 20 h, 2 h milled alloy) was very recently reported. ${ }^{12}$
In the present work we disclose the evolution of the magnetic behavior of the bulk material (AFM) to the SG behavior in 7 milled alloys, forming an ideal series to establish trends. This will be accomplished by selecting the (nano)particle size as the driving parameter. The article will present and discuss a structural characterization (Section III) followed by a macroscopic analysis of the static $\left(M_{D C}\right)$ and dynamic susceptibilities $\left(\chi_{A C}\right)$ (Section IV.A and IV.B). The microscopic understanding is supported by the magnetic Rietveld analysis conducted in low temperature neutron diffraction (ND) experiments, which are presented in Section IV.C. A magnetic phase diagram will be presented in the Discussion Section (IV). Finally, we summarize our main findings in the last section.

\section{EXPERIMENTAL DETAILS}

Polycrystalline samples of $\mathrm{TbCu}_{2}$ were alloyed in an arc furnace, under an Ar atmosphere, from stoichiometric amounts of $\mathrm{Tb}(3 \mathrm{~N})$ and $\mathrm{Cu}(5 \mathrm{~N})$ metals obtaining large quantities of alloy $(\approx 5 \mathrm{~g})$. Resulting metals were annealed for 5 days at $750{ }^{\circ} \mathrm{C}$, in vacuum $\left(\approx 10^{-3} \mathrm{mbar}\right)$ in order to improve the crystallinity and ensure a good homogenization of the samples. Later on, the samples were crushed and milled in a high-energy planetary mill, at a rotation speed of $200 \mathrm{rpm}$. The initial powders were placed in tungsten carbide containers using a ball/sample weight ratio 12:1. These containers were hermetically closed in the glove box in an $\operatorname{Ar}(99.99 \%)$ atmosphere to minimize the powder oxidation. The milling procedure was carried out following successive steps of 5 minutes of clockwise and anti-clockwise rotation with a 5 minutes intermediate stop.

Amounts of material were removed after certain milling times ( $t=0.5,1,2,3,5,10$, and 15 hours) to gather experimental data, as it will be explained in the following sections. X-ray diffraction (XRD) was performed at room temperature, using $\mathrm{Cu}-\mathrm{K}_{\alpha}(\lambda=1.5418 \AA)$ and Mo- $\mathrm{K}_{\alpha}(\lambda=0.7107 \AA)$ radiations. Transmission electron microscopy (TEM) was performed to check the nanocrystalline arrangement in a Jeol 2100 microscope. DC and AC magnetic susceptibility measurements were performed in a Quantum Design PPMS, thanks to the large $\mathrm{Tb}$-signal, in the temperature range 2-300 K. For the static DC magnetization, $H \leq 90 \mathrm{kOe}$ were used. AC-susceptibility data were obtained with an oscillating field $h=1$ Oe and frequencies $(f) 66 \mathrm{~Hz} \leq f \leq$ $10 \mathrm{kHz}$. Neutron diffraction spectra were collected at G4.1 instrument, located in Leon Brillouin Laboratory (France), using a wavelength $\lambda=2.423 \AA$ and at different temperatures between $1.5 \mathrm{~K}$ and $300 \mathrm{~K}$. The spectra were taken during 8 hours/temperature to get a high signal/noise ratio, with a remarkable amount of nanometric sample around $2 \mathrm{~g}$. 


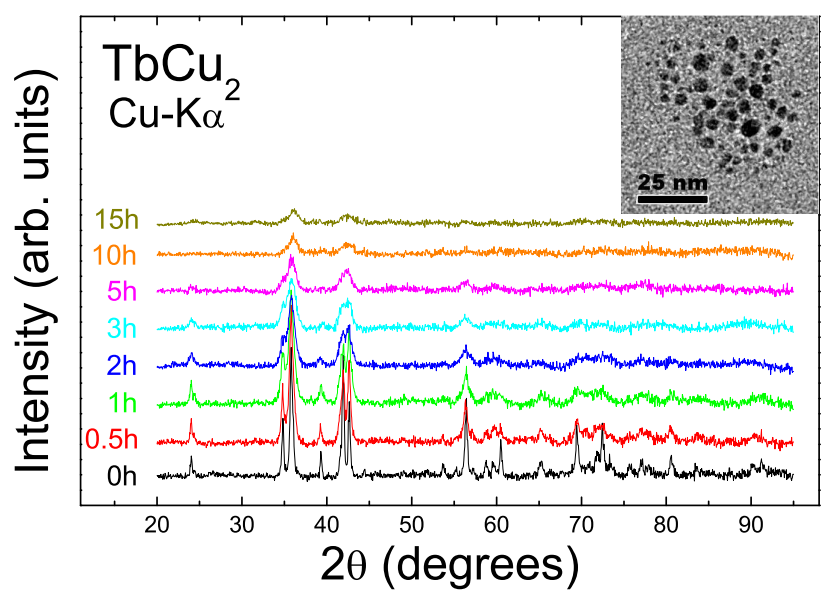

FIG. 1: (Color online) XRD patterns obtained with $\mathrm{Cu}-\mathrm{K}_{\alpha}$ radiation, for bulk $(0 \mathrm{~h})$ and milled $\mathrm{TbCu}_{2}$ alloys. Patterns have been shifted up for clarity. Inset: TEM image of a collection of nanoparticles in $t=10 \mathrm{~h}$ alloy

\section{STRUCTURE OF NANOPARTICLES}

XRD patterns are shown in Fig. 1. A broadening of the peaks is observed as milling time increases; a sign witnessing that $D$ decreases with longer grindings. ${ }^{20,24}$ The visualization of XRD patterns of Fig. 1 indicates that it is only reasonable to determine the particle size, from the $\mathrm{Cu}-\mathrm{K}_{\alpha} \mathrm{XRD}$, for $t \leq 5 h$, as for $t>5 \mathrm{~h}$ the patterns show very broad peaks and a halo due to the amorphization of $\mathrm{TbCu}_{2}$ appears between $30^{\circ}$ and $50^{\circ}$. Inset of Fig. 1 shows a representative TEM image of $t=10$ sample. Spherical NPs are visible and the size distribution gives $D_{T E M}=6.1(3) \mathrm{nm}$. The NPs distribute randomly, as was found for $t=2 \mathrm{~h}$ alloy. ${ }^{12}$ The XRD patterns have been refined by the Rietveld method (FULLPROF suite ${ }^{25}$ ) employing the resolution function with a $\mathrm{LaB}_{6}$ calibration. The fits were carried out using a Thomson-Cox-Hastings function, allowing the simultaneous calculation of particle size $(D)$ and strain $(\eta)$. Main structural parameters are summarized in Table I and show that very low milling times $(t<1 \mathrm{~h})$ to ensure nanometric particle sizes.

The Rietveld analysis of the diffractogram of the bulk alloy is consistent with a single phase of an orthorhombic $\mathrm{CeCu}_{2}$-type structure with unit cell parameters: $a=$ $4.3165(1) \AA, b=6.8323(3) \AA$ A and $c=7.3202(3) \stackrel{\circ}{A}$. These cell parameters are in very good agreement with those found in bulk $\mathrm{TbCu}_{2} \cdot{ }^{12,21}$ Atomic positions influencing the intensity of diffraction peaks are $\mathrm{Tb} 4 c$ and $\mathrm{Cu} 4 c$. The Rietveld refinement of the $1 \mathrm{~h}$ milled alloy is shown in Fig. 2, as a case example. The evolution of the lattice parameters (Table I) indicates that the volume increases around $1 \%$ for the $0.5 \mathrm{~h}$ milled alloy. Subsequently there appears a progressive decrease towards the bulk values as it were occurring a lattice metallurgical relaxation.

Although the change of the cell parameters with grind-

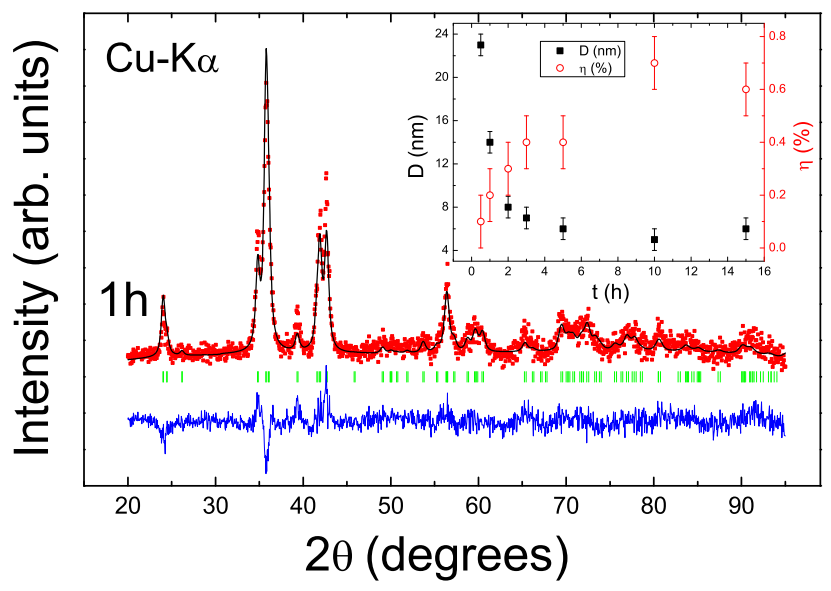

FIG. 2: (Color online) Rietveld refinement of the $\mathrm{Cu}-\mathrm{K}_{\alpha} \mathrm{XRD}$ pattern of the $1 \mathrm{~h}$ milled alloy (at $T=300 \mathrm{~K}$ ). The theoretical calculation is depicted as a continuous (black) line and the experimental data as points (in red). The (blue) line below the spectra represents the difference between the calculated and experimental patterns. Inset: Brisk particle size decrease $(D)$ with milling time. Single-domain nanoparticles are already obtained with only $t=1 \mathrm{~h}$. The microstrain $\eta$ increases as a result of milling.

ing time (Table I) is less than $0.5 \%$ at a local range, it is true that the polycrystalline state is affected by the grinding. This is deduced by considering the increasing values for $\eta$, reaching around $0.6 \%$ (see inset of Fig. 2). Such an increase is a typical result in milled alloys, and is a basis to demonstrate that some atoms become crystallographically disordered. ${ }^{21,24}$ Regarding the size variation, the milling process results in nanometric particles, as discussed in previous works. ${ }^{12,21}$ The $D(t)$ and $\eta(t)$ behaviors are similar to those observed in other $4 f-18,21$ and $3 d-\mathrm{Cu}^{26}$ based planetary-milled alloys, and the only most noticeable difference is, as it has been commented above, the reduced time required to achieve nanometric particle sizes. For example, $t=300$ hours for $D=14 \mathrm{~nm}$ $\mathrm{TbAl}_{2}{ }^{21}, t=120$ hours for $D=21 \mathrm{~nm} \mathrm{GdAl}{ }_{2}{ }^{18}$ and $t=$ 20 hours for $D=18 \mathrm{~nm} \mathrm{YbAl}_{3}{ }^{24}$, while for $\mathrm{TbCu}_{2}$ only $t=0.5 \mathrm{~h}$ were necessary to reduce particle size down to $D=23 \mathrm{~nm}$. It is clear to observe, in inset of Fig. 2, that the $D(t)$ curve is saturated with grinding time $(t \gtrsim$ $5 \mathrm{~h}$ ), and so, $\mathrm{TbCu}_{2}$ particles with $D \lesssim 5 \mathrm{~nm}$ cannot be obtained by planetary milling. In addition, the XRD patterns become less defined for $t \geq 5 \mathrm{~h}$, hampering the interpretation of results. Thus, in the following, we will pay more attention on the $t \leq 5 h$ alloys. 
TABLE I: Orthorhombic cell parameters $a, b$ and $c$; size $D$ and strain $\eta$ of particles at different milling times $(t)$, obtained by Rietveld refinement, with different wavelengths $\left(\mathrm{Cu}-\right.$ or $\mathrm{Mo}-\mathrm{K}_{\alpha}$ radiations), at room temperature. Standard Bragg errors are denoted by $R_{B}$.

\begin{tabular}{cccccccc}
\hline$t(h)$ & Radiation & $a(\AA)$ & $b(\AA)$ & $c(\AA)$ & $D(n m)$ & $\eta(\%)$ & $R_{B}(\%)$ \\
\hline 0 & $\mathrm{Cu}-\mathrm{K}_{\alpha}$ & $4.3165(1)$ & $6.8323(3)$ & $7.3202(3)$ & - & - & 23.1 \\
0.5 & $\mathrm{Cu}-\mathrm{K}_{\alpha}$ & $4.3292(5)$ & $6.8537(9)$ & $7.3332(9)$ & $23(1)$ & $0.1(1)$ & 20.5 \\
1 & $\mathrm{Cu}-\mathrm{K}_{\alpha}$ & $4.3237(7)$ & $6.846(1)$ & $7.319(1)$ & $14(1)$ & $0.2(1)$ & 16.1 \\
2 & $\mathrm{Cu}-\mathrm{K}_{\alpha}$ & $4.322(1)$ & $6.822(1)$ & $7.313(2)$ & $8(1)$ & $0.3(1)$ & 16.3 \\
3 & $\mathrm{Cu}-\mathrm{K}_{\alpha}$ & $4.318(2)$ & $6.835(3)$ & $7.329(4)$ & $7(1)$ & $0.4(1)$ & 21.5 \\
5 & $\mathrm{Cu}-\mathrm{K}_{\alpha}$ & $4.320(3)$ & $6.846(5)$ & $7.358(6)$ & $6(1)$ & $0.4(1)$ & 21.2 \\
10 & $\mathrm{Mo}-\mathrm{K}_{\alpha}$ & $4.306(2)$ & $6.863(2)$ & $7.391(3)$ & $5(1)$ & $0.7(1)$ & 10.8 \\
15 & $\mathrm{Mo}-\mathrm{K}_{\alpha}$ & $4.312(2)$ & $6.8600(2)$ & $7.327(3)$ & $6(1)$ & $0.6(1)$ & 18.0 \\
\hline \hline
\end{tabular}

\section{STATIC AND DYNAMIC MAGNETIC PROPERTIES}

\section{A. DC-magnetic susceptibility}

The zero field cooled (ZFC) curves of the DCsusceptibility $(M / H)$ at a magnetic field of $H=1000 \mathrm{Oe}$ show the AFM transition at around $50 \mathrm{~K}$, whose peak magnitude becomes reduced in the alloys subjected to longer milling times. In particular, for $t=15 \mathrm{~h}$, the peak is nearly absent (see Fig. 3). By contrast, the decrease of particle size gives rise to an enhanced SG transition around $15 \mathrm{~K}$. Its magnitude follows the opposite tendency to that just described for the $T_{N}$ peak. In consequence, both transitions are clearly correlated between each other and the particle size. FC curves were also recorded and, as an example, the $\mathrm{FC}$ variation is shown for $t=3 \mathrm{~h}$, to confirm the existence of irreversibility, typical of disordered magnets.

Lets us now focus on the surroundings of the AFM peak transition. Above the transition temperature $T_{N}$, the samples are in a paramagnetic state, where they obey a Curie-Weiss behavior (both insets of Fig. 3). The bulk sample has an effective moment of $10.04(1) \mu_{B}$ and a Curie-Weiss temperature $\theta_{p}=-7.2$ (2) K (therefore, antiferromagnetic). However, when we grind our sample for up to 15 hours, the Curie-Weiss temperature increases up to $\theta_{p}=11.1(2) \mathrm{K}$ (hence, no longer only antiferromagnetic) and the effective moment remains more or less the same $\left(\mu=9.92(1) \mu_{B}\right)$, close to the calculated (Hunds rules $\mathrm{Tb}, J=6)$ effective moment $\left(9.72 \mu_{B}\right){ }^{27}$ This is pointing out a tendency to favor slowly a positive exchange coupling.

The observation of Fig. 3 unveils the marked decrease of $T_{N}$ with decreasing particle size. For $D \sim 7 \mathrm{~nm}$, $\Delta T_{N} / T_{N} \cong-0.2$, which is an extremely large variation for the critical temperature. Effectively, in ferromagnetic $\mathrm{TbAl}_{2}$ nanometric alloys, $\Delta T_{C} / T_{C} \cong-0.06$ (reaching there $D \cong 14 \mathrm{~nm}$ ) which is an order of magnitude smaller. ${ }^{22}$ The variation of the ordering temperature with size is still relatively controversial. In $\mathrm{MnFe}_{2} \mathrm{O}_{4}$ ferrimagnetic NPs, $\Delta T_{C} / T_{C} \cong 0.02$, thus the $T_{C}$ increased with

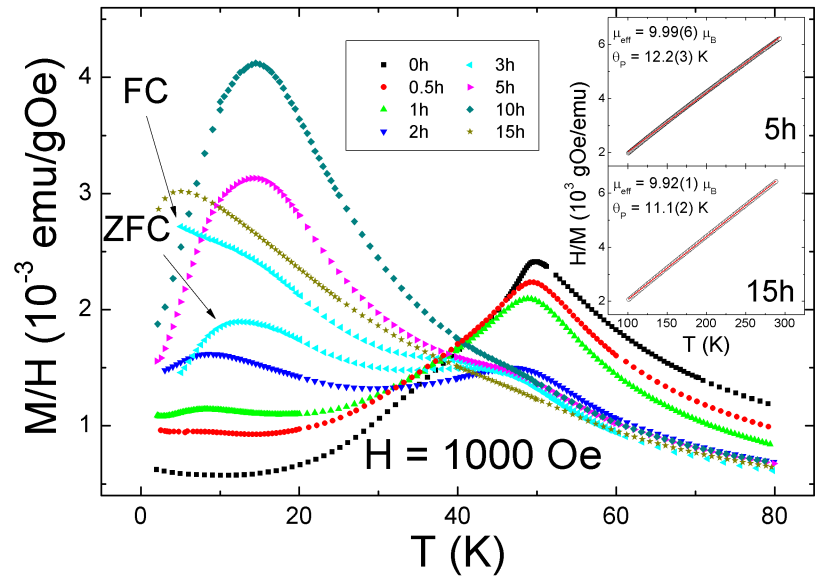

FIG. 3: (Color online) ZFC curves (DC-susceptibility) for the bulk and the milled samples, at $H=1000$ Oe. The FC curve is included for $t=3 \mathrm{~h}$ to observe the irreversibility. The evolution from AFM to SG can be observed; for the $t \geq 5 \mathrm{~h}$ milled alloys, almost no AFM signal is found. Inset: CurieWeiss behavior at $T>T_{N}$ for $t=5,15 \mathrm{~h}$.

decreasing size due to the interactions with the particle coating. ${ }^{28}$ Later it was reported that there is a $T_{C}(D)$ reduction, relative to the bulk, as size was decreased. ${ }^{29,30}$ Still, there are not many previous experimental reports as the ordering temperatures are frequently above room temperature.

Turning our attention to the low temperature freezing temperature, there is a $\Delta T_{f} / T_{f} \cong 0.28$. To investigate the possible size dependence, one can think of a powerlaw variation. A pure $T_{f} \propto D^{-1}$ dependence would reflect a surface/volume direct correlation, However, our value is different from exponent -1 and this would indicate the need for another factor acting in the freezing process. A sensible ingredient explaining that is the increase of interfacial effects among the NPs, provoking an enhancement of the magnetic spin disorder at the surface of the nearby particles.

In Fig. 4, the general $M_{D C}(H)$ behavior of the $\mathrm{TbCu}_{2}$ nanoparticles is examined. The data have been recorded 
up to $H=80 \mathrm{kOe}$. On the one hand, the metamagnetic transition present in the bulk alloy ${ }^{21,31}$ is progressively reduced with the milling, being wiped out for $t \geq$ $3 \mathrm{~h}$. This presence of the AFM state is confirmed by the variation of the Arrott plots (see bottom inset of Fig. 4), with a characteristic bending. Secondly, the magnetization value follows two tendencies: for $t<3$ $\mathrm{h}$, the $M(H=80 \mathrm{kOe})$ becomes reduced, but then for the longer milling time alloys, the magnetization tends to recover a larger value reaching around $M \cong 5 \mu_{B} / \mathrm{Tb}$. This recovery should be connected to the influence of a growing FM coupling at the interface. The lack of saturation deserves two comments: firstly there is an intrinsic high anisotropy in the bulk alloy ${ }^{31}$ and, secondly, it is a common finding in magnetically disordered materials (including magnetic nanoparticles) as a consequence of a surface moment canting. ${ }^{20,32,33}$

Now we focus on the $H_{C}(D)$ variation. Conventional AFM compounds should not display coercivity. Hence, in disordered systems, the competing AFM/FM interactions when passing across the freezing transition result in an intrinsic increase of the anisotropy and consequently of $H_{C} .{ }^{32}$ In the top inset of Fig. 4, the $H_{C}(D)$ is represented. The tendency is pristine with an increase of $H_{C}$ when reducing the particle size, reinforcing the fact that the coercivity found here is only due to a size reduction effect, independently of the intrinsic high anisotropy of bulk $\mathrm{TbCu}_{2} .{ }^{31}$ This fact can be understood by the relative higher contribution from a disordered shell which is randomized respect to the particle core and hence promoting a higher value of the anisotropy $K$. In ensembles of magnetic NPs, the value of $K$ is found to be size dependent. ${ }^{34,35}$ It should be reminded that in AFM NPs the interparticle interaction due to dipolar coupling is minimized and there might appear a very weak ferromagnetic component due to sublattice mismatch. The interparticle interaction affecting the effective anisotropy can be estimated as an effective temperature shifting up of the blocking temperature. ${ }^{1,13}$ In short, the increase of the magnetic disordered layer should be the major responsible for the increase of $H_{C}$ with decreasing $D$, observed here. Precisely, that disordered layer was causing the $H_{C}$ increase commented previously.

In nanogranular systems, it is a common feature to encounter a different atomic/magnetic arrangement in what it is widely known as core-shell structure. These two regions lead to some modifications in $H_{C}$ and in the hysteresis loop position. Fine particles were the first type of systems where this shift was discovered. ${ }^{36}$ The physical origin is the exchange anisotropy between (typically) adjacent FM and AFM layers. The most apparent macroscopic evidence is the mentioned loop shift and concomitant exchange bias (EB). ${ }^{37}$ Given that particles are of nanometric size, it is expected a slight difference of the magnetic behavior of the moments in the core and those at the surface. The surface shell may comprise a few magnetic layers of uncompensated moments. In spherical particles the shell thickness may vary around 0.6-1

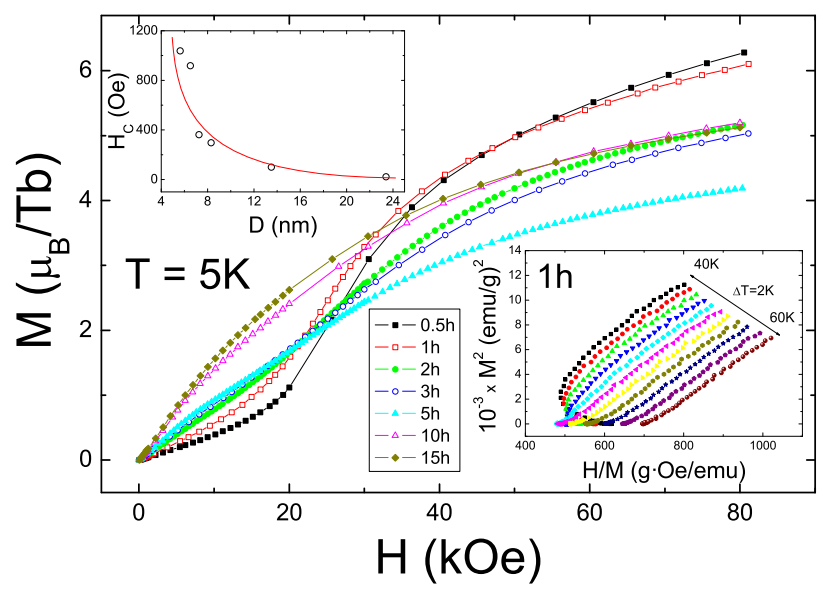

FIG. 4: (Color online) Field dependence of the isothermal magnetization of $\mathrm{TbCu}_{2}$ milled alloys at $T=5 \mathrm{~K}$. Top inset: coercive field dependence on the particle size for milling times $t \leq 10 \mathrm{~h}$. Bottom inset: Arrott plots of $t=1 \mathrm{~h}$ sample.

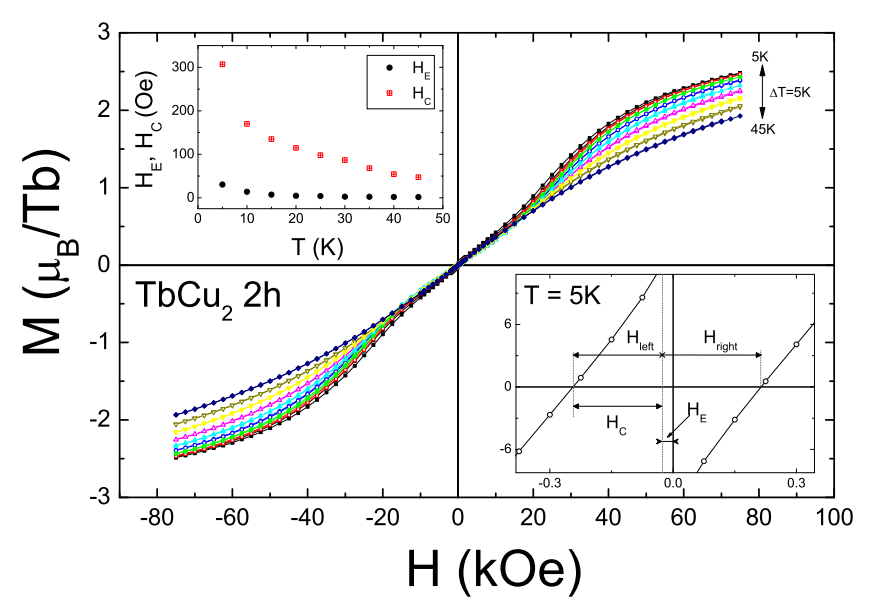

FIG. 5: (Color online) Exchange bias study of hysteresis loops of $\mathrm{TbCu}_{2}$ nanoparticles after a field cooling $(H=75 \mathrm{kOe})$ at several temperatures $\left(T<T_{N}\right)$ in $t=2 \mathrm{~h}$. The exchange bias, $H_{E}$, and the coercivity, $H_{C}$, are shown in the top inset. Lower inset: Zoom of the low field region, as an example.

nm. ${ }^{33}$ Very briefly, in a FC sequence there might appear a preferred orientation imposed by the anisotropy of the AFM. When the field is removed, the FM core suffers the field associated to the AFM resulting in the $\mathrm{EB}$ effect $^{37}$. The actual measurement involves to apply the maximum possible $H$ above the $T_{N}$ of the AFM regime. Then, the sample is cooled down to the lowest temperature and the loop is recorded. In order to elucidate the possible core-shell structure in our particles, we performed FC hysteresis loops. In Fig. 5 we show the hysteresis loops, at different temperatures $\left(T<T_{N}\right)$ of the milled alloy with magnetic fields up to $H=75$ $\mathrm{kOe}$, after the field cooling procedure. Values for the exchange anisotropy were obtained through conventional: $H_{E}=-\left(\left|H_{\text {left }}\right|+\left|H_{\text {right }}\right|\right) / 2$, with $H_{E}$, exchange bias 
field. Then, to better understand the complementary behavior of the coercive field $H_{C}(T)$ and $H_{E}(T)$ both of them are shown together in the upper inset of Fig. 5.

The results depicted in Fig. 5 indicate the existence of a negative loop shift (see lower inset). The absolute magnitude in $2 \mathrm{~h} \mathrm{TbCu}_{2}$ is small as $H_{C}(T=5 \mathrm{~K}) 300$ Oe and $H_{E}(T=5 \mathrm{~K}) 30$ Oe. For example, nanoparticles of $\mathrm{Co}-\mathrm{CoO}$ display $\mathrm{H}_{E}=9500 \mathrm{Oe}^{38}$ The EB effect with a $\mathrm{SG}$ surface $(0.6 \mathrm{~nm})$ layer has been found in $\mathrm{Fe}_{2} \mathrm{O}_{3}$ particles $(D=10 \mathrm{~nm}),{ }^{33}$ with much larger values $H_{C}(T=5 \mathrm{~K})=2000$ Oe and $H_{E}(T=5 \mathrm{~K})=1500$ Oe $)$ than the ones reported for the present alloy, and in $\mathrm{SG}$ amorphous FeZr. ${ }^{39}$ It is worth to remind that our characteristic temperatures $\left(T_{N}=46.8 \mathrm{~K}\right.$ and $\left.T_{f} 10 \mathrm{~K}\right)$ are very low, supporting the large difference in $H_{E}$ values in $\mathrm{TbCu}_{2}$ NPs.

A comment should be devoted to the increase of $H_{E}(T)$ towards low temperature. In nano $\gamma-\mathrm{Fe}_{2} \mathrm{O}_{3}$ where there is an EB effect caused by surface spins, it was noted the existence two linear regimes for $H_{E}(T)$, with an abrupt increase for (there labelled) $\mathrm{T}_{\text {crit }}>30 \mathrm{~K} .{ }^{33} \mathrm{In} 2 \mathrm{~h} \mathrm{TbCu}{ }_{2}$ the effect is still present with a much higher slope for $\mathrm{T}_{\text {crit }}$ $\leq 15 \mathrm{~K}$ (see upper inset Fig. 5). This is due to the fact that $H_{E}$ only exists while the spins on the surface of the particles are still frozen. The correlation between $H_{E}$ and $H_{C}$ is also known. Effectively, in the present work, there is an uprise in the $H_{C}$ below $\mathrm{T}_{f}=10 \mathrm{~K}$. This increase of $H_{C}$ is due to the energy that is required to switch the spins, pinned by the frozen-in spin glass layers. ${ }^{12}$ Care should be though taken into account as there are two factors altering the overall picture. The first one is that the $H_{E}$ increase is observed slightly above the $\mathrm{T}_{f}$; such a finding is a result of the breadth of the particle size distribution (affecting the magnetic strength of the shell too). On the other hand, the lack of a clear kink at $\mathrm{T}_{\text {crit }}$ and possibly the low magnitude of $H_{E}$ are a combined outcome of the existence of some interparticle magnetic coupling through the SG interface.

\section{B. AC-magnetic susceptibility}

The spin dynamics is studied in a QD-PPMS magnetometer using a oscillating field $h=1$ Oe, thanks to the considerable signal of the $\mathrm{TbCu}_{2}(\approx 40 \mathrm{mg})$ samples. The frequency $f$ was varied between $66 \mathrm{~Hz}$ and $10 \mathrm{KHz}$. In Fig. 6, the real- $\left(\chi^{\prime}(T)\right)$ and complex- $\left(\chi^{\prime \prime}(T)\right)$ components of the AC-susceptibility for $t=1,3,5,10$ and 15 hours milled $\mathrm{TbCu}_{2}$ alloys at $\omega / 2 \pi=1 \mathrm{kHz}$ are plotted as a function of temperature.

The shape of the $\chi^{\prime}(T)$ curves is similar to that observed in DC-susceptibility (ZFC curves): an AFM transition that disappears with milling, leading to a SG behavior at low temperatures, reinforced as particle size is lowered. In the case of $\chi^{\prime \prime}(T)$, for $t \geq 3 h$, the Néel transition is nearly absent, marking that AFM is disappearing favoring an overall spin-glass state within the alloy, while the low $\mathrm{T}_{f}$ peak is clearly observable The AC-

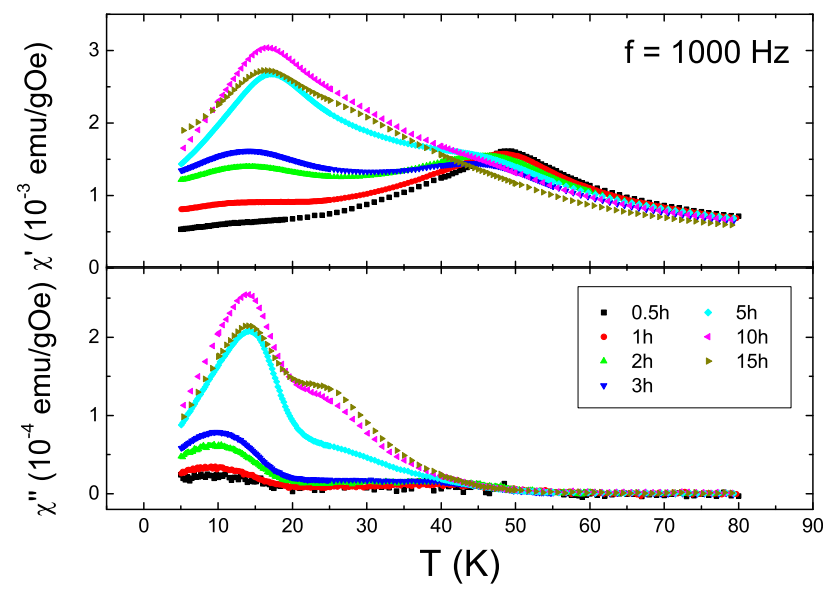

FIG. 6: (Color online) In-phase $\left(\chi^{\prime}\right)$ and out-phase $\left(\chi^{\prime \prime}\right)$ components of the AC-susceptibility for different milling times, for $f=1000 \mathrm{~Hz}(h=1 \mathrm{Oe})$. As milling time increases, AFM disappears favoring a SG arrangement; but when $t \simeq 10 \mathrm{~h} \mathrm{a}$ contribution at $T \simeq 25 \mathrm{~K}$ begins to appear.

susceptibility measurements (see Fig. 7) confirm the displacement of $T_{N}$ to lower temperatures and $T_{f}$ to higher temperatures with increasing milling times, which was observed and thoroughly discussed using the $M_{D C}(H, T)$. Besides, a seminal report in $\mathrm{GdAl}_{2}$ also finds a similar displacement in a member of the $R X_{2}$ family. ${ }^{18}$ In our nano- $\mathrm{TbCu}_{2}$, with the increase of the milling time, a hump appears, only visible in the $\chi^{\prime \prime}(T)$ (Fig. 6), for $t \geq 5 \mathrm{~h}$ at around $T \cong 25 \mathrm{~K}$. This weak contribution is not visible either in DC-susceptibility, as it can be observed in Fig. 3. Despite the commented structural fact that for the $t=5,10,15 \mathrm{~h}$ samples the XRD data become less and less undefined (see Fig. 1), it is possible to propose an interpretation for the observed shoulder: the Néel temperature is practically absent, meaning that the AFM-coupled particles are being, somehow, solved in the metallic (magnetically disordered) environment. In this situation, it is plausible that the now clustered, rather than nanoparticle, state triggers the upraise of FM-coupled moments. This would give rise to an energy absorption and thus the mentioned shoulder. Precisely, we remind that $\theta_{P}>0$ for $t=15 \mathrm{~h}$ in the Curie-Weiss analysis, and the commented recovery of the magnetization in the $\mathrm{M}(\mathrm{H})$ curves shown in Fig. 5, both backing up the emerging FM-coupling at the interface.

In Fig. 7, the frequency-dependent $\chi^{\prime}(T), \chi^{\prime \prime}(T)$, are depicted for $t=5 \mathrm{~h}$. The two transition temperatures shift (i) to slightly lower temperatures in the case of $T_{N}$ (with an almost negligible change) and (ii) to higher temperatures in the case of $T_{f}$, with increasing frequencies. Moreover, the latter peak in $\chi^{\prime}(T)$ at $T_{f}$ decreases in magnitude with higher frequencies. In the following we are proceeding to explain a quantitative analysis of the spin dynamics of three characteristic samples. A fast (and simple) evaluation is to calculate the freezing temperature shift with frequency. This is commonly carried 


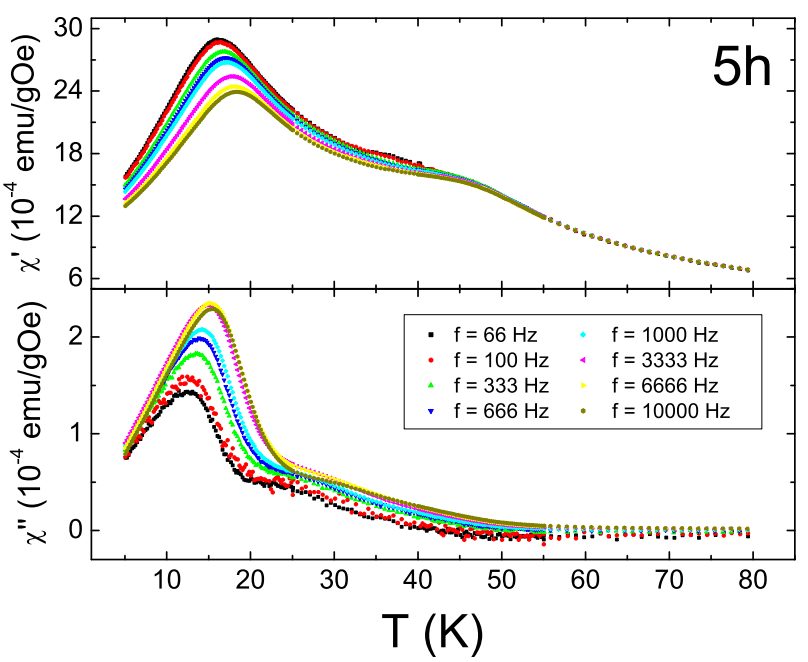

FIG. 7: (Color online) Dynamic susceptibility variation with frequency for $t=5 \mathrm{~h}$ nano- $\mathrm{TbCu}_{2}$, as a representative example. In-phase $\left(\chi^{\prime}\right)$ and out-phase $\left(\chi^{\prime \prime}\right)$ components of the AC-susceptibility show a clear shift to higher temperatures with increasing $f$ for the freezing temperature (low $T$ peak) whereas no modification is observed in $T_{N}$ (high $T$ hump).

out by using the $\delta$-shift parameter as:

$$
\delta=\frac{\Delta T_{f}}{T_{f} \Delta \log \omega} \Rightarrow \log \omega=\frac{1}{\delta} \ln T_{f}+\text { constant }
$$

where $\omega=2 \pi f$. If the magnetic interparticle interactions are absent (or negligible), the spin particle rotation under the uniaxial anisotropy is understood by a thermal activation process, following the well-known Arrhenius-Néel law and for a typical $\chi_{A C}$ measurement $(f=10-10000$ $\mathrm{Hz})$ in a non-interacting $\mathrm{SPM}$ system $\left(\tau_{0} \approx 10^{-9} \mathrm{~s}\right), \delta=$ $0.13-0.24$, as commonly accepted. ${ }^{40}$ Whenever a reduction of such $\delta$-values is found, the nanoparticles become magnetically correlated and, in the limit, a cluster glass behavior should control the spin divergence at the critical temperature. Finally, the $\delta$-shift is practically inexistent in canonical SG involving the freezing of individual spins $\left(\delta=0.0045\right.$ for $\left.\mathrm{AuMn}^{32}\right)$ and negligible in phase transitions $\left(T_{C}, T_{N}\right)$. The $\delta$-values obtained from the fit to Eq. 1 for every milled alloy are displayed in Table II. In our particular case, $\delta=0.02-0.06$ for the different milled alloys, higher than spin glasses ${ }^{32}$ ) but lower than cluster glasses $\left(\mathrm{Fe}_{91} \mathrm{Zr}_{9}, \delta=0.066{ }^{41}\right)$ As the nano- $\mathrm{TbCu}_{2} \delta$ values are higher than those for ideal SGs, the magnetic spin relaxation is expected to take place at a slower rate.

Firstly we start checking out the divergent spin correlation at $T_{f}$. An order parameter $q_{E A}$ was defined by Edwards and Anderson; ${ }^{42}$ this was taken as the mean-square spontaneous magnetization in a single energy minimum, averaged to all possible minima values. In other words, the average value of the spin-spin autocorrelation function. In the vicinity of the freezing temperature, the spin fluctuations will follow critical fluctuations. At temperatures around the freezing temperature, a spin-spin cor-
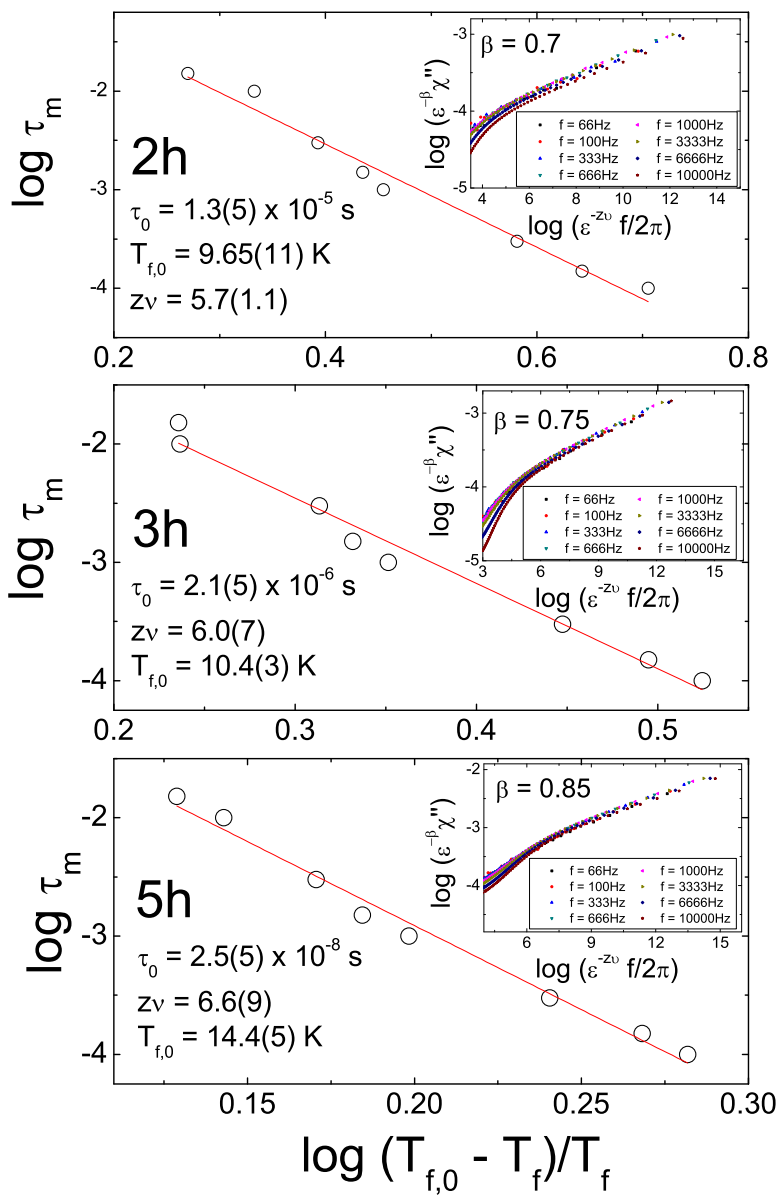

FIG. 8: (Color online) Critical slowing down for several milled $\mathrm{TbCu}_{2}$ alloys. Inset: Collapsing curves to extract the $\beta$ exponent according to Eq. (4).

relation length, shorter than the coherence length of the critical fluctuations, will be controlled precisely by the latter. Thus, the coherence length can be easily transformed into timescales in a conventional critical slowing down, giving

$$
\tau_{m}=\tau_{0}\left(\frac{T_{f, 0}-T_{f}}{T_{f}}\right)^{-z v}
$$

where $\tau_{m}$ is the measurement time, $\tau_{0}$ is the relaxation time of an individual particle moment at a $f \rightarrow 0, z v$ is the dynamic critical exponent and $T_{f, 0}$ is the static spin glass temperature. In addition, it is convenient to analyze $\chi$ " $(T)$ at several frequencies to extract the $\beta$ dynamic exponent with the scaling:

$$
\chi^{\prime \prime}(f / 2 \pi, T)=\epsilon^{\beta} F[(f / 2 \pi) \epsilon]^{z v}
$$

where $\epsilon=\left(T-T_{f}\right) / T_{f}$. Reasonable parameters can be obtained from the fit of the $t=2,3,5 \mathrm{~h}$ data (see graphical results in Fig. 8), and are inserted in Table 
TABLE II: Parameter values of $\delta, \tau_{0}, \mathrm{z} \nu, \beta$, and $\mathrm{T}_{f}, 0$ for characteristic milling times $t(\mathrm{~h})$.

\begin{tabular}{cccccc}
\hline \hline$t(\mathrm{~h})$ & $\delta$ & $\tau_{0}(\mathrm{~s})$ & $z \nu$ & $\beta$ & $T_{f, 0}(\mathrm{~K})$ \\
\hline 2 & $0.056(3)$ & $1.3(5) \times 10^{-5}$ & $5.7(3)$ & $0.70(5)$ & $9.65(11)$ \\
3 & $0.044(2)$ & $2.1(5) \times 10^{-6}$ & $6.0(7)$ & $0.75(5)$ & $10.4(3)$ \\
5 & $0.025(1)$ & $2.5(5) \times 10^{-8}$ & $6.6(9)$ & $0.85(5)$ & $14.4(5)$ \\
\hline \hline
\end{tabular}

II. The value of $\tau_{0}$ for lower milling times $(t=2,3 \mathrm{~h})$ is higher than expected for SGs, whereas for milling times $t$ $=5 \mathrm{~h}$ closer values of $\tau_{0}$ for SGs are achieved, with $\tau_{0} \sim$ $10^{-8}-10^{-13} \mathrm{~s}^{32}$ The $t<5 \mathrm{~h}$ large values of $\tau_{0}$ are related to the fact that our systems follow a relaxation rate of the nanoparticles slightly slower than in conventional SGs, as already explained in the former $\delta$ description. Indeed, high a $\tau_{0}$ has already been observed in FeC interacting nanoparticle ferrofluids $\left(\tau_{0} \sim 10^{-6} \mathrm{~s}\right) .{ }^{43}$ The eventual modification of $\tau_{0}$ may be slightly temperature-influenced and also dependent on the reduced damping constant associated to the flipping rate. ${ }^{44}$ and by the existence of interactions, with a modification of the SPM energy barrier. ${ }^{45}$

The results in Table II indicate that the $z \nu$ values are within the range of SGs, $z \nu=4-13 .^{8,10}$ Moreover, Fig. 8 also depicts the collapsing curves of the dynamic scaling of the complex component following Eq. (3). The observation of such figures show that the scaling is improved for the $t=5 \mathrm{~h}$ sample $(\beta=0.85)$. To understand better these values, we have inserted a selection of values of magnetically disordered materials in Fig. 9. The choice of the materials looks for a representative character: they vary from the structural point of view, including amorphous alloys (i.e. $\mathrm{Fe}_{91} \mathrm{Zr}_{9}{ }^{41}$ ), crystalline $\left(\left(\mathrm{MnF}_{2}\right)_{0.65}\left(\mathrm{BaF}_{2}\right)_{0.15}\left(\mathrm{NaPO}_{3}\right)_{0.20}{ }^{46}\right)$, quasi-amorphous $\left(\mathrm{Fe}_{30} \mathrm{Ag}_{40} \mathrm{~W}_{30}\right)^{47}$, well defined nanoparticles (Fe-C), ${ }^{43}$ multilayers $\left(\mathrm{Co}_{80} \mathrm{Fe}_{20} / \mathrm{Al}_{2} \mathrm{O}_{3}{ }^{48}\right)$ There are oxides such as $\left(\mathrm{LaPb}(\mathrm{Mn}, \mathrm{Fe}) \mathrm{O}_{3},{ }^{49} \mathrm{LaCaMnO}_{3},{ }^{50}\right)$ and different metals $\left(\mathrm{CeNi}_{0.5} \mathrm{Cu}_{0.5},{ }^{51} \mathrm{U}_{1-x} \mathrm{Y}_{x} \mathrm{Pd}_{3},{ }^{52} \mathrm{Ag} M n,{ }^{53} \mathrm{Fe}_{70} \mathrm{Al}_{30},{ }^{54}\right.$ $\mathrm{CuMn},{ }^{55} \mathrm{Al}_{24} \mathrm{Cu}_{70} \mathrm{Mn}_{6} .{ }^{56}$ ) have been inserted as well. Bearing in mind the definition of the nature of the SG transition, it is possible to suggest some boundaries which will be helpful to understand the global behavior. These are the (green) dashed lines marking the regions for SG, CSG and SSG states. These are not perfectly defined regions as disordered magnetism is a progressive change of the magnetic spin correlation coupling, preventing the existence of defined limits.

Another dynamic aspect that may render valuable information is the analysis Argand diagrams at a certain temperature which are related to the energy barrier distribution. If single-relaxation processes are followed by the flipping spins, the complex susceptibility is described by the Casimir-du Pré equation

$$
\chi=\chi(\infty)+\frac{\chi(0)-\chi(\infty)}{1-i \omega \tau}
$$

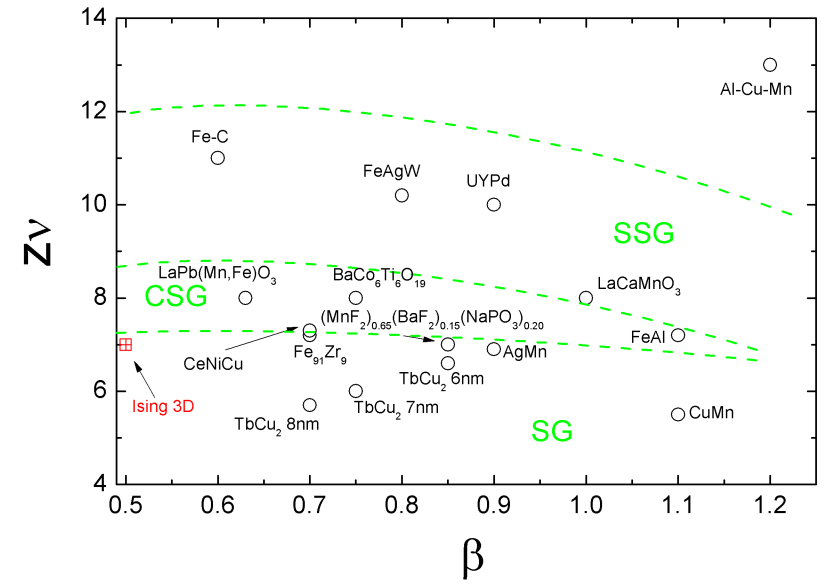

FIG. 9: (Color online) Overview of magnetically disordered compounds depending on their $z \nu$ and $\beta$ critical exponents. Data have been taken from several references (see text).
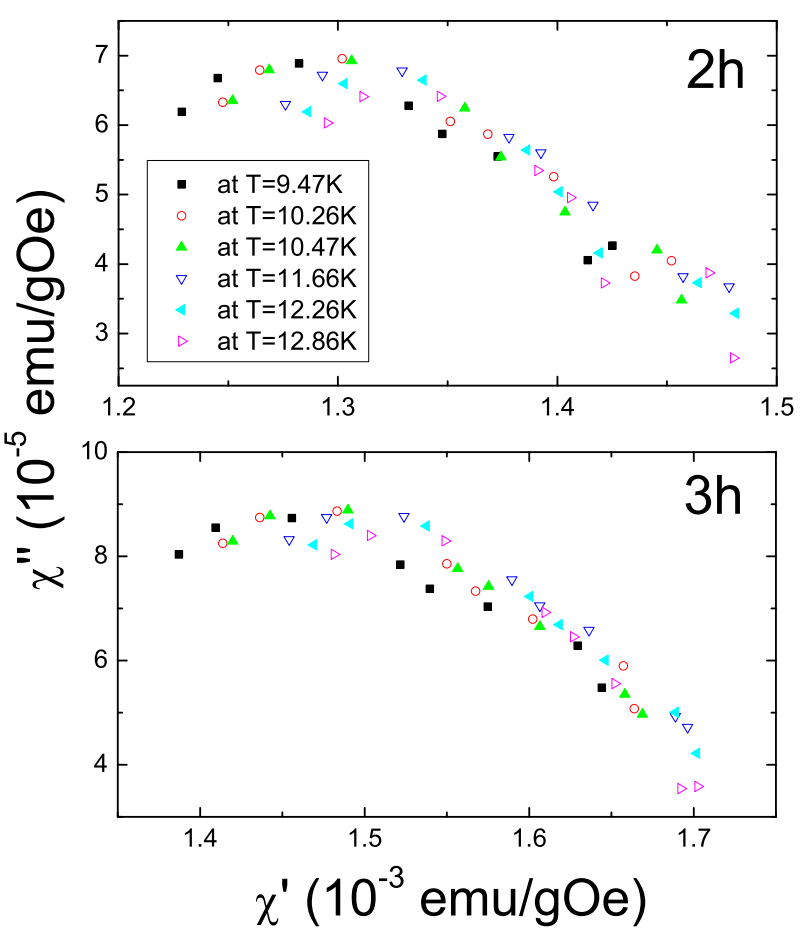

FIG. 10: (Color online) Argand diagrams around the freezing transition for nano- $\mathrm{TbCu}_{2}$ milled during $t=2 \mathrm{~h}$ and $t=3 \mathrm{~h}$.

In the case of an ideal SPM system, a half-circle is found when plotting $\chi$ "vs. $\chi^{\prime}$ for the different $f$-values at a given $T{ }^{8}$ If Argand diagrams for different temperatures collapse onto a single curve, then the energy barrier is independent of the temperature. Deviations from the half-circle would serve as an estimation for the energy distribution caused by the size distribution or the dipole-dipole interaction. ${ }^{57}$ Nevertheless, for supermagnetic systems, it has been proposed that the semicircle 
should appear modified as a consequence of the interparticle couplings. In that situation, the Argand representation, including a distribution of relaxation times, can exhibit the fingerprints of several dynamic modes of domain walls. ${ }^{8}$ The supporting assumption for this is the necessary coupling among the NPs which are then resembling the conventional domains in a ferromagnet. In this sense, Argand plots for SFM $\left[\mathrm{CoFe}(1.4 \mathrm{~nm}) / \mathrm{Al}_{2} \mathrm{O}_{3}(3\right.$ $\mathrm{nm})]_{10}$ provided an experimental evidence of the domain modes. ${ }^{58}$ In Fig. 10 the $\chi$ " vs. $\chi^{\prime}$ plots of the milled alloys $(t=2,3 \mathrm{~h})$ are presented. These are far from being a perfect half-circle, as expected in these supermagnetic systems. and we observe that there exists a maximum of every plot. In SG systems it has also been claimed that a broad distribution of relaxation times causes a flattening of the curves, ${ }^{32}$ which is the case in the present nano$\mathrm{TbCu}_{2}$. In any case, the inspection of the width of the distribution taken from the $\chi^{\prime \prime}$ maximum value is slightly larger $\left(\cong 0.2510^{-3} \mathrm{emu} / \mathrm{gOe}\right)$ for $t=3 \mathrm{~h}$ than for $t=$ $2 \mathrm{~h}\left(\cong 0.1510^{-3} \mathrm{emu} / \mathrm{gOe}\right)$. Thereby, this is indicating the enhancement of the SG contribution in that alloy as well.

\section{Neutron diffraction}

Microscopic magnetic structure analyses were performed in the milled samples, showing straightforward results of prominent value the final appraisal of the magnetic behavior of nanometric $\mathrm{TbCu}_{2}$. Neutron diffraction (ND) patterns were taken at different temperatures: 1.5 $\mathrm{K}$ and $10 \mathrm{~K}\left(T<T_{f}\right), 25 \mathrm{~K}$ and $36 \mathrm{~K}\left(\mathrm{~T}_{f}<T<T_{N}\right)$ and $60 \mathrm{~K}$ and $300 \mathrm{~K}\left(T>T_{N}\right)$, and shown for $5 \mathrm{~h}$ milled alloy at $T=1.5 \mathrm{~K}$ in Fig. 11 .

The magnetic structure corresponds to a collinear commensurate AFM with two propagation vectors $q_{1}=$ $(0,0,0)$ and $q_{2}=(1 / 3,0,0)$, as previously reported in bulk and $2 \mathrm{~h}$ milled sample. ${ }^{12,23}$ Thus, the magnetic structure is not modified with respect to that of the bulk alloy. The resulting ordered $\mathrm{Tb}^{3+}$ magnetic moment, at $T=1.5 \mathrm{~K}$, is $\mu=7.2(2) \mu_{B}$. The magnetic size is also calculated and gives $D_{M A G}=8(1) \mathrm{nm}$, which is very close to the one in $2 \mathrm{~h}$ sample. This means that the particle core is single domain and a unique magnetic ordering is maintained with respect to the bulk. The thermal variation (relative to $T_{N}$ ) of the magnetic moment is presented in the inset of Fig.11(a). In this, the variation follows roughly that of a Brillouin curve with $J=6$ (see continuous line). There is a reduction (20\%) of the neutron magnetic intensity, at $T=1.5 \mathrm{~K}$, with respect to the (neutron) bulk, $\mu=8.8 \mu_{B}$, revealing a disordered environment (magnetic moment canting), affected by the decrease of surface coordination in relation with that of the atoms within the particles. An estimation of the ratio of the surface atoms $\left(N_{S}\right)$ respect to those in the total volume $\left(N_{V}\right)$ is given by

$$
N_{S} / N_{V}=(3 \varsigma / D)
$$
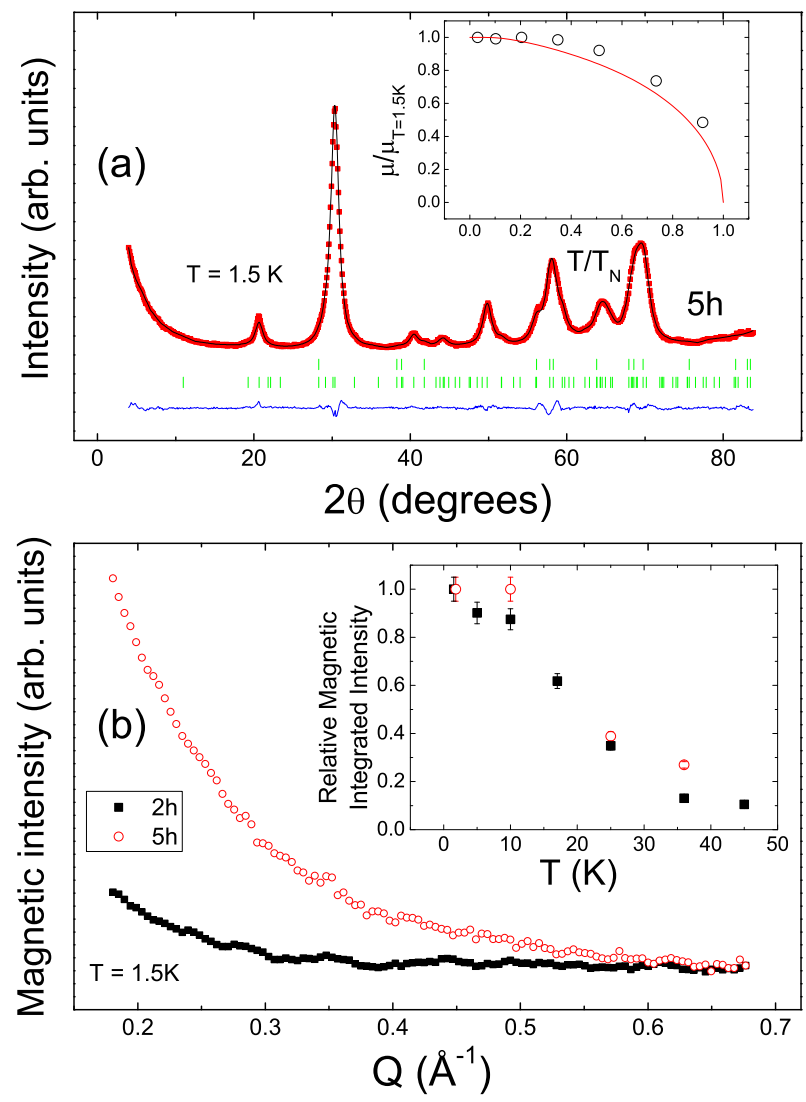

FIG. 11: (Color online) (a): Nuclear and magnetic contributions for the $5 \mathrm{~h} \mathrm{TbCu}_{2}$ milled alloy (at $T=1.5 \mathrm{~K}$ ). Vertical marks correspond to the position of the allowed Bragg reflections. The blue line corresponds to the difference between the observed (red dots) and the calculated (black line) intensity, by Rietveld refinement. Inset (a): Thermal variation of $\mathrm{Tb}$ magnetic moment. The continuous line is a calculation of the Brillouin function with $J=6(\mathrm{~Tb})$.(b): Variation of the magnetic intensity in the low $Q$-region at $T=1.5 \mathrm{~K}$. Inset (b): Relative variation of the integrated intensity for $Q<0.7 \AA^{-1}$.

where $\varsigma$ is the thickness of the grain boundary. ${ }^{59}$ The resulting value is $40 \%$. This is indicating again the role of the surface moments, and hence supporting the surface moment reduction. Such a reduction is also apparent in the ND of other AFM NPs. ${ }^{14,15}$ In short, the AFM coupling is retained in the $\mathrm{TbCu}_{2}$ single-domain particles, whose moment becomes reduced approaching the Néel transition. In Fig. 11(b), it is also noted that low-angle $\left(2 \theta<15\right.$, i.e.: $\left.Q<0.7 \AA^{-1}\right)$ signal increases markedly, as was detected in the $2 \mathrm{~h}$ case. ${ }^{12}$ In the $t=5 \mathrm{~h}$ case the increase is even more marked. Clearly there is a consistent magnetic coupling among some of the single domain particles, which is enhanced if the interface is disordered. The thermal variation of such a low- $Q$ increase is very similar in both alloys, as visible in inset of Fig. 11(b). 


\section{DISCUSSION}

The high-energy milling process of bulk $\mathrm{TbCu}_{2}$ results in the production of randomly grouped ensembles of particles with a nanometric size distribution. Inside each particle, the orthorhombic Imma symmetry is retained (see Table I). The particle size decreases as the milling time becomes longer, reaching a saturation $D \cong 6 \mathrm{~nm}$. On the other hand, particle strain increases with milling time, up to $\eta \cong 0.6 \%$, for the $15 \mathrm{~h} \mathrm{TbCu} 2$.In this final discussion we will address two distinct issues. Initially we will consider the macroscopic implications of nano$\mathrm{TbCu}_{2}$ as an overall magnetic response to compare with the effects of the chemical substitution in bulk $R X_{2}$ systems. Then, we will deepen in the analysis of properties from a nanoscopic standpoint to put forward an attractive magnetic phase diagram.

In bulk form, it is feasible to prepare pseudobinary alloys. In this sense, the evidences from the dilution of the parent alloy with non-magnetic Y-atoms, as $\mathrm{Tb}_{x} \mathrm{Y}_{1-x} \mathrm{Cu}_{2}$, indicates a weakening of the magnetic interaction reducing the $T_{N}$. This reduction follows the empirical relation $T_{N}(x) / T_{N}(x=1) \propto\left(x-x_{c}\right)^{0.6}$, where $x_{c}$ is the critical concentration at which the magnetic ordering disappears. ${ }^{60}$ In our case, the reduction $\Delta T_{N} / T_{N, \text { bulk }} \simeq-11 \%$ resulting in a $x \sim 0.97$ (a minute dilution) which suggests that the antiferromagnetic order is not essentially altered. Another via for compositional dilution is that established by the exchange of $\mathrm{Cu}$ by $\mathrm{Ni}$ atoms; with this, the electron concentration, is hence altered. In the case of $\mathrm{Tb}(\mathrm{Ni}, \mathrm{Cu})_{2}$ such a replacement increases the AFM character and eventually gives rise to a $\mathrm{FM}(\sim 8 \% \mathrm{Ni})$ structure. $^{31}$ In our nano-TbCu , we observe the disappearance of the AFM order which is not substituted by long-range ferromagnetism here, but by a magnetically disordered state. In addition our reduction is much smaller than that found for the former cases and we ought to reconcile that observation with another explanation. This requirement is reinforced by the evident correlation between the $T_{N}$ and $T_{f}$ values in the NPs.

At this point it is possible to render a nanoscopic point of view. In view of the size-induced variation of key temperatures, we propose a useful magnetic phase diagram (see Fig. 12). The size variation provides the basic understanding of the magnetic states present in the alloys and the effect of the strain is more apparent in those alloys with high milling times affecting the magnetic response of the disordered shell, which may become a connected intergrain boundary. This is especially apparent in the variation field-dependent Magnetization (Fig. 4) and the values of dynamic exponents (Fig. 8). Overall, this magnetic phase diagram is similar to those of other magnetically disordered systems (so-called reentrant magnets), but driven mainly by the size instead of the concentration of the magnetic ions. ${ }^{32}$ In the case of bulk $R E$-alloys there are also examples of magnetic reentrance, as occurs in the weak random-axis magnets, with Laves-phase structure $^{61}$ and in amorphous magnets as $\mathrm{Dy}_{6} \mathrm{Fe}_{74} \mathrm{~B}_{20}$,

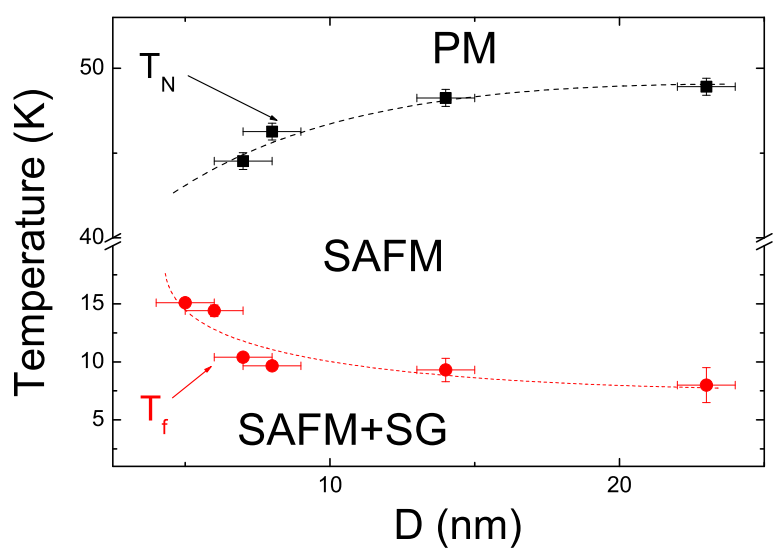

FIG. 12: (Color online) Magnetic phase diagram of $\mathrm{TbCu}_{2}$ nanomagnets with characteristic Néel and freezing temperatures. Dashed lines are guides for the eye.

with a behavior explained according to the onset of an easy axis which varies following the local arrangement (short-range structure). ${ }^{62} \mathrm{~A}$ more general interpretation of coexistence of ordered and disordered magnetism is given by the Sherrington-Kirkpatrick model, as a result of the replica symmetry breaking. ${ }^{63}$ Another more recent analytical approach employs small clusters with AFM order producing a SG state, but still clearly of smaller size than our NPs. ${ }^{64}$

Regarding the SAFM state, this arrangement is connected with those reported in other supermagnetic systems. Nevertheless most of them have been worked out in superferromagnetic/super spin glass state $9,10,65,66$. The latter scenario is very close to the one in milled alloys of $\mathrm{GdAl}_{2}{ }^{18}$ and $\mathrm{TbAl}_{2}{ }^{20,22}$ for which the ferromagnetic transition was disappearing at the expense of a growing SG state. In fact, this idea of definite coupling among nanoparticles was first proposed in antiferromagnetic goethite $\alpha$-FeOOH. ${ }^{67}$ Very recently it has been shown that goethite properties are dominated by the AFM order, followed by a lower transition in the fine particle state, and a further very low temperature magnetic coupling mode. ${ }^{68,69}$ In essence, this Fe oxihydroxide turns out as a macroscopically very similar system to nano- $\mathrm{TbCu}_{2}$. Therefore, the study of SAFM $R$-based nanoparticles allow to extract information on the surface moments given that the particle cores have a negligible response as reported in Fe-oxides. ${ }^{33,70}$. The proposal of new magnetic phase diagrams in AFM NPs will also be required for the cases in which the disordered shell, when the particles are very small, becomes predominant. ${ }^{71}$ 


\section{CONCLUSIONS}

The evolution of the magnetic properties in a nanoparticle system $\mathrm{TbCu}_{2}$ as function of the milling time (particle size) has been characterized by comprehensive magnetic susceptibility and neutron diffraction analyses. A full picture with a magnetic phase diagram as function of the particle size is proposed. The release of such a magnetic phase diagram driven by size-variations constitutes a milestone in the understanding of the behavior of nanosized AFM rare earth systems. Effectively, it immediately resembles the diagrams of magnetically disordered systems, especially those of the so-called reentrant magnets. Here, nano- $\mathrm{TbCu}_{2}$ constitutes an inspiring metallic system in which the size evolution triggers a macroscopic coexistence of (super)antiferromagnetic and spin glass. In addition we have detected that the spin canting is governed by the reduction of particle size and a the exis- tence of microstructural strain, which both are enhancing an interfacial disordered coupling between particles.

\section{ACKNOWLEDGEMENTS}

This work has been supported by a CICYT MAT201127573-C04 grant. The experiment at LLB was supported by the European Commission through the Access Activities of the Integrated Infrastructure Initiative for Neutron Scattering and Muon Spectroscopy (NMI3), supported by the European Commission under the $7^{\text {th }}$ Framework Programme through the Key Action: Strengthening the European Research Area, Research Infrastructures, Contract NMI3-II/FP7 $\mathrm{N}^{\circ}$ 283883. LFB would like to valuable comments by Sergio Magalhães (U. Fluminense, Brazil).
1 J. L. Dormann, D. Fiorani, and E. Tronc, Adv. Chem. Phys. 97, 283 (1997).

2 S. Mørup, M. F. Hansen, and C. Frandsen, in Comprehensive Nanoscience and Nanotechnology, Vol. 1 (Oxford Academic Press, 2011) p. 437.

${ }^{3}$ Q. A. Pankhurst, J. Connolly, S. K. Jones, and J. Dobson, J. Phys. D 36, R167 (2003); Q. A. Pankhurst, N. T. K. Thanh, S. K. Jones and J. Dobson, J. Phys. D 42, 224001 (2009).

4 Samuel C.N. Tang, Irene M.C. Lo, Water Research 47, 2613 (2013).

${ }^{5}$ S. Bader, Rev. Mod. Phys. 78, 1 (2006).

6 An-Hui Lu, E. L. Salabas, and Ferdi Schüth, Angew. Chem. 46, 1222 (2007).

7 M. Zhang, X. Tie, M. Tang, C. S. Criddle, Y. Cui, and S. X. Wang, Nature Commun. 4, 2892 (2013).

8 S. Bedanta and W. Kleemann, J. Phys. D 42, 13001 (2009).

9 S. Bedanta, T. Eimüller, W. Kleemann, J. Rhensius, F. Stromberg, E. Amaladass, S. Cardoso, and P. P. Freitas, Phys. Rev. Lett. 98, 176601 (2007).

10 J. Alonso, M. L. Fdez-Gubieda, J. M. Barandiarán, A. Svalov, L. Fernández Barquín, D. Alba Venero, and I. Orue, Phys. Rev. B 82, 054406 (2010).

11 Sayan Chandra, H. Khurshid, Wanfeng Li, G. C. Hadjipanayis, M. H. Phan, and H. Srikanth, Phys. Rev. B 86, 014426 (2012).

12 C. Echevarria-Bonet, D. P. Rojas, J. I. Espeso, J. Rodríguez Fernández, L. Rodríguez Fernández, P. Gorria, J. A. Blanco, M. L. Fdez-Gubieda, E. Bauer, G. André, and L. Fernández Barquín, Phys. Rev. B 87 180407(R) (2013).

13 S. Mørup, D. E. Madsen, C. Frandsen, C. R. H. Bahl, and M. F. Hansen, J. Phys.: Condens. Matter 19, 213202 (2007).

14 C. H. Wang, S. N. Baker, M. D. Lumsden, S. E. Nagler, W. T. Heller, G. A. Baker, P. D. Deen, L. M. D. Cranswick, Y. Su, and A. D. Christianson, Phys. Rev. B 83, 214418 (2011).

15 N. J. O. Silva, M. Karmaoui, V. S. Amaral, I. PuenteOrench, J. Campo, I. da Silva, A. Ibarra, R. Bustamante,
A. Millán, and F. Palacio, Phys. Rev. B 87, 224429 (2013).

16 Oliver Gutfleisch, Matthew A. Willard, Ekkes Brück, Christina H. Chen, S. G. Sankar and J. Ping Liu, Adv. Mat. 23, 821 (2011).

17 N. H. Luong and J. Franse, Magnetic properties of Rare Earth-Cu $\mathrm{u}_{2}$ compounds in Handbook of Magnetic Materials (K. H. J. Buschow, ed., Elsevier), vol. 8, p. 415 (1995).

18 G. F. Zhou and H. Bakker, Phys. Rev. B 52, 9437 (1995).

19 M. A. Morales, D. S. Williams, P. M. Shand, C. Stark, T. M. Pekarek, L. P. Yue, V. Petkov, and D. L. Leslie-Pelecky, Phys. Rev. B 70184407 (2004).

20 D. P. Rojas, L. Fernández Barquín, J. Rodríguez Fernández, J. I. Espeso and J. C. Gómez Sal, J. Phys.: Condens. Matter 19, 186214 (2007).

21 D. P. Rojas, J. I. Espeso, L. Fernández Barquín, J. Rodríguez Fernández, and J. C. Gómez Sal, J. Magn. Magn. Mater. 310, e506 (2007).

22 D. P. Rojas, L. Fernández Barquín, C. Echevarria-Bonet, J. Rodríguez Fernández, J. Nanosci. Nanotech. 12, 7482 (2012).

23 V. Sima, Z. Smetana, B. Lebech, and E. Gratz, J. Magn. Magn. Mater. 54-57, 1357 (1986).

24 D. P. Rojas, L. Fernández Barquín, J. I. Espeso, J. Rodríguez Fernández, and J. Chaboy, Phys. Rev. B 78, 094412 (2008).

25 J. Rodriguez-Carvajal, Physica B 192, 55 (1993).

${ }^{26}$ M. Azabou, L. Escoda, J. J. Suñol, and M. Khitouni, EPJ Web of Conferences 29, 00048 (2012).

27 N. Ashcroft and N. Mermin, Solid State Physics (Saunders College, Philadelphia, 1976).

28 Z. X. Tang, C. M. Sorensen, K. J. Klabunde, and G. C. Hadjipanayis, Phys. Rev. Lett. 67, 3602 (1991).

29 J. P. Chen, C. M. Sorensen, K. J. Klabunde, G. C. Hadjipanayis, E. Devlin, and A. Kostikas, Phys. Rev. B, 54, 9288 (1996).

30 Jun Wang, Wei Wu, Fan Zhao, and Guo-meng Zhao, Appl. Phys. Lett. 98, 083107 (2011).

31 C. A. Poldy and E. Gratz, J. Magn. Magn. Mat. 8, 223 (1978).

32 J. A. Mydosh, Spin glasses: An experimental Introduction 
(Taylor \& Francis, London, 1993).

33 B. Martinez, X. Obradors, L. Balcells, A. Rouanet, and C. Monty, Phys. Rev. Lett. 80, 181 (1998).

${ }^{34}$ F. Luis, J. M. Torres, L. M. García, J. Bartolomé, J. Stankiewicz, F. Petroff, F. Fettar, J.-L. Maurice, and A. Vaurès, Phys. Rev B 65, 094409 (2002).

${ }^{35}$ L. Fernández Barquín, R. García Calderón, B. Farago, J. Rodríguez-Carvajal, A. Bleloch, D. McComb, R. Chater, and Q. A. Pankhurst, Phys. Rev. B 76, 172404 (2007).

36 W. H. Meiklejohn and C. P. Bean, Phys. Rev. 105, 904 (1957).

37 J. Nogués and I. K. Schuller, J. Magn. Magn. Mat. 192, 203 (1999).

38 S. Gangopadhyay, G. C. Hadjipanayis, C. M. Sorensen, and K. J. Klabunde, J. Appl. Phys. 73, 6964 (1993).

39 H. Morita, H. Hiroyoshi, K. Fukamichi, J. Phys. F 16, 507 (1986).

40 D. H. Ucko, Q. A. Pankhurst, L. Fernández Barquín, J. Rodríguez Fernández, and S. F. J. Cox, Phys. Rev. B 64, 104433 (2001).

${ }^{41}$ L. Fernández Barquín, J. C. Gómez Sal, P. Gorria, J. Garitaonandia, and J. M. Barandiarán, Eur. Phys. Journal B 35, 3 (2003).

42 S. F. Edwards and P. W. Anderson, J. Phys F: Met. Phys. 5, 965 (1975).

43 C. Djurberg, P. Svedlindh, P. Nordblad, M. F. Hansen, F. Bodker, and S. Mørup, Phys. Rev. Lett. 79, 5154 (1997).

${ }^{44}$ W. T. Coffey, D. S. F. Crothers, Y. P. Kalmykov, E. S. Massawe, and J. T. Waldron, J. Magn. Magn. Mater. 127, L254 (1993).

45 J. L. Dormann, F. D’Orazio, F. Lucari, E. Tronc, P. Prené, J. P. Jolivet, D. Fiorani, R. Cherkaoui, and M. Noguès, Phys. Rev. B 53, 14291 (1996).

46 P. Beauvillain, J. P. Renard, M. Matecki, and J. J. Prejean, Europhys. Lett. 2, 23 (1986).

47 J. A. de Toro, M. A. Lopez de la Torre, J. M. Riveiro, A. Beesley, J. P. Goff, and M. F. Thomas, Phys. Rev. B 69, 224407 (2004).

48 S. Sahoo, O. Petracic, C. Binek, W. Kleemann, J. B. Sousa, S. Cardoso, and P. P. Freitas, Phys. Rev. B 65, 134406 (2002).

49 Y. Bitla, S. N. Kaul, and L. Fernández Barquín, Phys. Rev. B 86, 094405 (2012).

50 M. Perovic, V. Kusigerski, V. Spasojevic, A. Mrakovic, J. Blanusa, M. Zentkova, and M. Mihalik, J. Phys. D: Appl. Phys. 46, 165001 (2013).

51 N. Marcano, J. C. Gómez Sal, J. I. Espeso, L. Fernández Barquín, and C. Paulsen, Phys. Rev. B 76, 224419 (2007).

${ }^{52}$ M. A. Lopez de la Torre, J. Rodríguez Fernández, K. A. McEwen, and M. B. Maple, Phys. Rev. B 74, 014431 (2006).

53 L. P. Levy and A. T. Ogielski, Phys. Rev. Lett. 57, 3288 (1986).
${ }^{54}$ D. Alba Venero, R. García Calderón, L. Fernández Barquín, E. Apiñaniz, J. S. Garitaonandia, and F. Plazaola, IEEE Trans. Magn, 44, 3883 (2008).

55 B. Barbara, A. P. Malozemoff, and Y. Imry, Phys. Rev. Lett. 47, 1852 (1981).

56 E. Obradó, A. Planes, and B. Martínez, Phys. Rev. B 59, 11450 (1999).

57 X. X. Zhang, Magnetic relaxation and quantum tunneling of magnetization, in Handbook of Advanced Magnetic Materials (Springer, 2006) p. 147

58 X. Chen, O. Sichelschmidt, W. Kleemann, O. Petracic, C. Binek, J. B. Sousa, S. Cardoso, and P. P. Freitas, Phys. Rev. Lett. 89, 137203 (2002).

59 D. P. Rojas, L. Fernández Barquín, J. Rodríguez Fernández, L. Rodríguez Fernández, J González, Nanotechnology 21, 445702 (2010).

60 Y. Hashimoto, H. Fujii, H. Fujiwara, and T. Okamoto, J. Phys. Soc. Japan 47, 67 (1979).

61 A. del Moral, J. I. Arnaudas, P. M. Gehring, M. B. Salamon, C. Ritter, E. Joven, and J. Cullen, Phys. Rev. B 47, 7892 (1993).

62 J. Tejada, B. Martinez, A. Labarta, and E. M. Chudnovsky, Phys. Rev. B 44, 7698 (1991).

63 D. Sherrington and S. Kirkpatrick, Phys. Rev. Lett. 35, 1792 (1975).

${ }^{64}$ C. F. Silva, F. M. Zimmer, S. G. Magalhaes, and C. Lacroix, Phys. Rev. E 86, 051104 (2012); F. M. Zimmer, M. Schmidt, and S. G. Magalhaes, Phys. Rev. E 89, 062117 (2014).

${ }^{65}$ J. A. De Toro, S. S. Lee, D. Salazar, J. L. Cheong, P. S. Normile, P. Muniz, J. M. Riveiro, M. Hillenkamp, F. Tournus, A. Tamion, and P. Nordblad, Appl. Phys. Lett. 102, 183104 (2013).

66 D. Alba Venero, L. Fernández Barquín,, J. Alonso, M. L. Fdez-Gubieda, L. Rodríguez Fernández, R. Boada, and J. Chaboy, J. Phys.: Condens. Matter, 25, 276001 (2013).

67 S. Mørup, M. B. Madsen, J. Franck, J. Villadsen, and C. J. W. Koch, J. Magn. Magn. Mater. 40, 163 (1983).

${ }^{68}$ Q. A. Pankhurst, L. Fernández Barquín, J. S. Lord, A. Amato, and U. Zimmermann, Phys. Rev. B 85, 174437 (2012).

69 E. Brok, C. Frandsen, D. E. Madsen, H. Jacobsen, J. O. Birk, K. Lefmann, J. Bendix, K. S. Pedersen, C. B. Boothroyd, A. A. Berhe, G. G. Simeoni, S. Mørup, J. Phys. D: Appl. Phys. 47, 365003 (2014).

70 Q. A. Pankhurst and R. J. Pollard, Phys. Rev. Lett., 67, 248 (1991).

${ }^{71}$ N. Rinaldi-Montes, P. Gorria, D. Martínez-Blanco, A. B. Fuertes, L. Fernández Barquín, J. Rodríguez Fernández, I. de Pedro, M. L. Fdez-Gubieda, J. Alonso, L. Olivi, G. Aquilanti, and J. A. Blanco, Nanoscale 6, 457 (2014). 